\title{
Revisiting the single-energy CT calibration for proton therapy treatment planning: a critical look at the stoichiometric method
}

\author{
Carles Gomà ${ }^{1,2}$, Isabel P Almeida ${ }^{3}$ and Frank Verhaegen ${ }^{3}$ \\ ${ }^{1}$ KU Leuven, Department of Oncology, Laboratory of Experimental Radiotherapy, \\ Leuven, Belgium. \\ ${ }^{2}$ University Hospitals Leuven, Department of Radiation Oncology, Leuven, Belgium. \\ ${ }^{3}$ Department of Radiation Oncology (MAASTRO), GROW - School for Oncology \\ and Developmental Biology, Maastricht University Medical Centre, Maastricht, The \\ Netherlands \\ E-mail: carles.goma@kuleuven.be
}

\begin{abstract}
Despite extensive research in dual-energy computed tomography (CT), single-energy CT (SECT) is still the standard imaging modality in proton therapy treatment planning and, in this context, the stoichiometric calibration method is considered to be the most accurate to establish a relationship between $\mathrm{CT}$ numbers and proton stopping power. This work revisits the SECT calibration for proton therapy treatment planning, with special emphasis on the stoichiometric method. Three different sets of tissue-substitutes of known elemental composition (Gammex, CIRS and Catphan) were scanned with the same scanning protocol. A stoichiometric fit was performed for each set of tissue-substitutes. Based on that, the CT number, relative electron density and relative proton stopping power were calculated for ICRU 46 biological tissues and the different sets of tissue-substitutes. Despite common belief, it was found that the stoichiometric fit depends on the elemental composition of the tissue-substitutes used in the calibration, leading to differences in relative stopping power up to $3.5 \%$ for cortical bone. In addition, according to Rutherford et al (1976 Neuroradiology 11 15-21) parametrization of the atomic cross-section, CT numbers of Gammex tissue-substitutes and ICRU 46 biological tissues were found to be similar within the whole energy range relevant to computed tomography. Consequently, it was found that, for Gammex tissue-substitutes, the CT calibration curve resulting from the stoichiometric method agrees with that obtained by simple interpolation of experimental data. In conclusion, the stoichiometric method for SECT calibration seems to depend on the tissue-substitutes used for calibration-which could be regarded as an additional source of uncertainty in proton range for bone tissues. Furthermore, Gammex tissue-substitutes appear to be a good representative of biological tissues within the energy range relevant to computed tomography - making the stoichiometric method unnecessary.
\end{abstract}




\section{Introduction}

Analytical dose calculation algorithms for proton beams need a computed tomography (CT) calibration curve that establishes the relationship between the CT number of a given voxel and its proton electronic stopping power (Seltzer et al. 2011), relative to the one of water - also called relative stopping power (RSP). Despite the increasing interest in the use of dual-energy CT (DECT) for proton therapy treatment planning, commercial treatment planning systems do not yet allow for a fully-integrated RSP calculation based on two CT numbers (per voxel) obtained at different $\mathrm{kVp}$. In other words, DECT information cannot yet be used without a pre-processing of the image by the user, with all the safety concerns that such a manipulation may raise. That is, current treatment planning systems only support single-energy CT (SECT) data and CT voxels are typically assigned a RSP value by means of a HU-to-RSP look-up table (LUT). A major problem of this approach is that, whereas one aims at a CT calibration curve which is accurate for biological tissues (i.e. in patient), RSP measurements of human (or animal) tissue samples are rather impractical, so particle therapy centres typically resort to tissue-substitutes to determine their CT calibration curves experimentally (Taasti et al. 2018).

To circumvent this problem, Schneider et al. (1996) proposed the stoichiometric calibration method. In brief, this method consists in characterizing the CT scanner indirectly (the energy spectrum of the x-ray source and the energy response of the radiation detector combined) by means of $\mathrm{CT}$ scans of tissue-substitutes, and afterwards predicting the $\mathrm{CT}$ numbers of biological tissues using this characterization. According to the authors, "It should be noted that these tissue substitutes do not necessarily have to be very tissue-like. It is possible to choose e.g. Lucite, Teflon, Delrin etc." (Schneider et al. 1996). That is, within reasonable limits, the method was claimed to be independent of the stoichiometric composition of the tissue-substitutes used to characterize the CT

scanner. Despite initial criticism (De Kock \& Schreuder 1996), the stoichiometric calibration is - with more than 700 citations $\ddagger$ - one of the most widely used methods to generate CT calibration curves from SECT data (Taasti et al. 2018) and, thus, it is often used as reference to assess the potential benefit of DECT over SECT in proton therapy treatment planning (Zhu \& Penfold 2016, Bär et al. 2017, Michalak et al. 2017, Bär et al. 2018b, Möhler et al. 2018, Xie et al. 2018).

This works revisits the SECT calibration for proton therapy treatment planning and shows that: (i) the stoichiometric calibration depends on the tissue-substitutes used in the CT characterization, introducing an additional source of systematic uncertainty in proton range (for bone tissues only), and (ii) depending on the tissue-substitutes chosen for CT characterization, the stoichiometric method becomes unnecessary, and it may be replaced by a much simpler method presented below.

$\ddagger$ https://scholar.google.com/ Last visited: October 29, 2018 


\section{Materials and methods}

\subsection{Theoretical evaluation of tissue-substitutes}

Three different sets of tissue-substitutes, from three different vendors-Gammex (Gammex Inc., Middleton, WI), CIRS (CIRS Inc., Norfolk, VA) and Catphan (The Phantom Laboratory, Salem, NY) - were investigated. The elemental composition and mass density of all tissue-substitutes studied theoretically in this work are tabulated in table 1, as reported to us by the different manufacturers. Although Catphan materials should not be considered tissue-equivalent (Verhaegen \& Devic 2005), in what follows we will refer to them as 'tissue-substitutes' for the sake of simplicity. As reference biological tissues, we chose the 50 adult biological tissues reported in ICRU 46 (White et al. 1992).

For all these tissues (both biological tissues and tissue-substitutes) we calculated the relative electron density (A.4), the relative stopping power (A.19), $\mathcal{Z}_{\mathrm{R}}$ and $\mathcal{Z}_{\mathrm{p}}$ (A.11). The relative stopping power was computed for a proton kinetic energy of $100 \mathrm{MeV}$ and a mean excitation energy of water of $I_{\mathrm{w}}=78 \mathrm{eV}$, based on the latest ICRU 90 recommendations (Seltzer et al. 2016). For biological tissues and tissue-substitutes, the $I$-value was calculated using the Bragg additivity rule and the $I$-values of the atomic constituents in solid state (Berger et al. 1984). Finally, the CT numbers based on two x-ray spectra at 80 and $140 \mathrm{kVp}$ (van der Heyden et al. 2018) were calculated using (A.15) and (A.16) - the energy response of the radiation detector was assumed to be constant. To that end, $k_{\mathrm{R}}(E)$ and $k_{\mathrm{p}}(E)$ were calculated using Rutherford et al. (1976) parametrization (A.8), and $k_{\mathrm{C}}(E)$, i.e. the electronic cross section for Compton scattering, was taken from ICRU 46 for liquid water and assumed to be constant for all biological tissues and tissue surrogates.

\subsection{Experimental evaluation of the stoichiometric method}

Irrespective of how close tissue-substitutes are to biological tissues, the stoichiometric method was claimed to perform well even with not "very tissue-like" tissue-substitutes, such as "Lucite, Teflon, Delrin etc." (Schneider et al. 1996).

In order to test this statement, three different 'electron density CT phantoms' (Gammex RMI 467, CIRS 062M and Catphan 504) with different tissue-substitutes were studied. The elemental composition and mass density of all the tissue-substitutes used experimentally in this study are tabulated in table 1 . The three CT phantoms were scanned in the same CT scanner (Sensation Open, Siemens Healthineers, Forchheim, Germany) using the exact same scanning protocol $(120 \mathrm{kVp}, 300 \mathrm{mAs}$, B30s reconstruction kernel, $400 \mathrm{~mm}$ field of view, $3 \mathrm{~mm}$ slice thickness). The centre of the phantoms was positioned at the isocentre of the $\mathrm{CT}$ scanner and no dose modulation was used. The mean CT numbers of each tissue-substitute were measured on the 5 central slices of the phantom and, together with their standard deviation, are reported in table 1. The CT number of air was measured without phantom.

Once both the elemental composition and the CT number of each tissue-substitute 
Table 1: Weight fraction, mass density, experimental HU and standard deviation $\left(\sigma_{\mathrm{HU}}\right)$ of different tissue-substitutes. The tissuesubstitutes marked with an asterisk $\left(^{\star}\right)$ were only used in the theoretical study and therefore do not have experimental HU and $\sigma_{\mathrm{HU}}$ values.

\begin{tabular}{|c|c|c|c|c|c|c|c|c|c|c|c|c|c|c|c|}
\hline $\begin{array}{l}\text { Element } \\
Z \\
A \\
I(\mathrm{eV})\end{array}$ & $\begin{array}{r}\mathrm{H} \\
1 \\
1.008 \\
19.2\end{array}$ & $\begin{array}{r}\mathrm{C} \\
6 \\
12.01 \\
81\end{array}$ & $\begin{array}{r}\mathrm{N} \\
7 \\
14.01 \\
82\end{array}$ & $\begin{array}{r}\mathrm{O} \\
8 \\
16.00 \\
106\end{array}$ & $\begin{array}{r}\mathrm{F} \\
9 \\
19.00 \\
112\end{array}$ & $\begin{array}{r}\mathrm{Mg} \\
12 \\
24.31 \\
176\end{array}$ & $\begin{array}{r}\mathrm{Si} \\
14 \\
28.09 \\
195\end{array}$ & $\begin{array}{r}\mathrm{P} \\
15 \\
30.97 \\
195\end{array}$ & $\begin{array}{r}\mathrm{S} \\
16 \\
32.06 \\
203\end{array}$ & $\begin{array}{r}\mathrm{Cl} \\
17 \\
35.45 \\
180\end{array}$ & $\begin{array}{r}\mathrm{Ca} \\
20 \\
40.08 \\
216\end{array}$ & $\begin{array}{r}\mathrm{Ba} \\
56 \\
137.3 \\
555\end{array}$ & $\rho\left(\mathrm{g} / \mathrm{cm}^{3}\right)$ & $\mathrm{HU}$ & $\sigma_{\mathrm{HU}}$ \\
\hline Lung (LN-300) \#455 & 8.46 & 59.38 & 1.96 & 18.14 & & 11.19 & 0.78 & & & 0.10 & & & 0.28 & -716 & 17 \\
\hline Lung (LN-450) \#485 & 8.47 & 59.57 & 1.97 & 18.11 & & 11.21 & 0.58 & & & 0.10 & & & 0.40 & -543 & 18 \\
\hline Adipose (AP6) \#453 & 9.06 & 72.30 & 2.25 & 16.27 & & & & & & 0.13 & & & 0.942 & -97 & 14 \\
\hline Breast (BR 12) \#454 & 8.59 & 70.11 & 2.33 & 17.90 & & & & & & 0.13 & 0.95 & & 0.977 & -58 & 13 \\
\hline Solid Water \#457 & 8.02 & 67.23 & 2.41 & 19.91 & & & & & & 0.14 & 2.31 & & 1.018 & -10 & 13 \\
\hline Muscle \#452* & 8.10 & 67.17 & 2.42 & 19.85 & & & & & & 0.14 & 2.32 & & 1.050 & - & - \\
\hline Brain (SR2) \#481 & 10.83 & 72.54 & 1.69 & 14.86 & & & & & & 0.08 & & & 1.053 & 15 & 12 \\
\hline Liver (LV1) \#482 & 8.06 & 67.01 & 2.47 & 20.01 & & & & & & 0.14 & 2.31 & & 1.097 & 68 & 14 \\
\hline Inner bone (IB3) \#456 & 6.67 & 55.64 & 1.96 & 23.52 & & & & 3.23 & & 0.11 & 8.86 & & 1.143 & 207 & 16 \\
\hline Bone Mineral (B200) \#487 & 6.65 & 55.52 & 1.98 & 23.64 & & & & 3.24 & & 0.11 & 8.87 & & 1.154 & 212 & 16 \\
\hline Bone $\left(\mathrm{CB} 2-10 \% \mathrm{CaCO}_{3}\right) \# 483$ * & 8.59 & 65.33 & 2.73 & 19.22 & & & & & & 0.14 & 4.00 & & 1.170 & - & - \\
\hline Bone $\left(\mathrm{CB} 2-30 \% \mathrm{CaCO}_{3}\right) \# 484$ & 6.68 & 53.48 & 2.12 & 25.61 & & & & & & 0.11 & 12.01 & & 1.335 & 440 & 17 \\
\hline Bone $\left(\mathrm{CB} 2-50 \% \mathrm{CaCO}_{3}\right) \# 480$ & 4.77 & 41.63 & 1.52 & 32.00 & & & & & & 0.08 & 20.02 & & 1.560 & 800 & 18 \\
\hline Cortical Bone (SB3) \#450 & 3.41 & 31.41 & 1.84 & 36.50 & & & & & & 0.04 & 26.81 & & 1.825 & 1202 & 21 \\
\hline \multicolumn{16}{|l|}{ CIRS } \\
\hline Adipose & 10.0 & 71.3 & 1.8 & 16.4 & & & & & & 0.2 & 0.3 & & 0.960 & -62 & 14 \\
\hline Breast 50/50 & 9.6 & 70.3 & 1.9 & 17.0 & & & & & & 0.2 & 0.9 & & 0.991 & -29 & 14 \\
\hline Plastic Water * & 7.9 & 53.6 & 1.7 & 27.2 & & 9.3 & & & & 0.23 & & & 1.029 & - & - \\
\hline Muscle & 9.1 & 69.7 & 2.1 & 16.8 & & & & & & 0.1 & 2.2 & & 1.062 & 51 & 13 \\
\hline Liver & 9.0 & 69.4 & 2.1 & 17.1 & & & & & & 0.1 & 2.2 & & 1.072 & 50 & 14 \\
\hline Bone $200 \mathrm{mg} / \mathrm{cc} \mathrm{HA}$ & 7.0 & 56.3 & 2.0 & 22.7 & & & & 3.3 & & 0.2 & 8.5 & & 1.161 & 215 & 13 \\
\hline Bone 800 mg/cc HA & 5.7 & 40.8 & 1.0 & 25.9 & & & & 8.3 & 0.07 & 0.03 & 17.9 & 0.28 & 1.530 & 817 & 20 \\
\hline Bone $1000 \mathrm{mg} / \mathrm{cc} \mathrm{HA}$ * & 4.5 & 35.4 & 1.2 & 29.4 & & & & 9.2 & 0.08 & 0.04 & 19.9 & 0.33 & 1.66 & - & - \\
\hline Bone 1250 mg/cc HA & 3.6 & 28.8 & 1.1 & 32.0 & & & & 10.8 & 0.08 & 0.04 & 23.3 & 0.32 & 1.82 & 1251 & 19 \\
\hline Bone $1500 \mathrm{mg} / \mathrm{cc} \mathrm{HA}$ * & 2.9 & 22.9 & 0.9 & 33.9 & & & & 12.4 & 0.06 & 0.03 & 26.7 & 0.26 & 1.99 & - & - \\
\hline Bone $1750 \mathrm{mg} / \mathrm{cc} \mathrm{HA}$ * & 2.3 & 17.9 & 0.7 & 35.6 & & & & 13.7 & 0.05 & 0.02 & 29.6 & 0.20 & 2.15 & - & - \\
\hline \multicolumn{16}{|l|}{ Catphan } \\
\hline PMP & 14.37 & 85.63 & & & & & & & & & & & 0.83 & -196 & 6 \\
\hline LDPE & 14.37 & 85.63 & & & & & & & & & & & 0.92 & -103 & 6 \\
\hline Polystyrene & 7.74 & 92.26 & & & & & & & & & & & 1.05 & -44 & 6 \\
\hline Acrylic & 8.05 & 59.98 & & 31.96 & & & & & & & & & 1.18 & 117 & 6 \\
\hline Delrin (POM) & 6.71 & 40.00 & & 53.28 & & & & & & & & & 1.41 & 331 & 6 \\
\hline Teflon & & 24.02 & & & 75.98 & & & & & & & & 2.16 & 957 & 12 \\
\hline
\end{tabular}


were known, we performed a separate stoichiometric fit (A.22) for each set of tissuesubstitutes. The elemental composition and CT number of air (HU $=-1002 \pm 5)$ was also used in all stoichiometric fits. The search space of possible $k$-values $\left(k_{1}, k_{2}\right)$ was limited to those with a physical meaning within the energy range of 30-150 keV (Jackson \& Hawkes 1981), based on Rutherford et al. (1976) parametrization-i.e. $2 \cdot 10^{-4}<k_{1}<6 \cdot 10^{-3}$ and $3 \cdot 10^{-6}<k_{2}<6 \cdot 10^{-4}$. If such constraints were not set, physically meaningless $k$-values were obtained for some of the phantoms (De Kock \& Schreuder 1996). In addition, in order to double-check our implementation of the stoichiometric fit, the experimental data in Schneider et al. (2000) was input, yielding the exact same $k$-values as reported therein.

Once the $k$-values were determined, the CT numbers of the reference ICRU 46 biological tissues and the three different sets of tissue-substitutes (Gammex, CIRS and Catphan) were calculated using (A.16) and (A.20). The uncertainty in the calculated CT numbers was estimated using the delta method, as described in Appendix B. The relative electron density and relative stopping power were also calculated using (A.4) and (A.19), respectively. The uncertainty in the relative stopping power, due to the uncertainty in the $I$-values, was not estimated. This point is a field of research by itself (De Smet et al. 2018, Bär et al. 2018a) and it falls beyond the scope of this work.

Finally, in order to confirm our results, different photon spectra (80, 100 and 140 $\mathrm{kVp}$ ) were also investigated. Furthermore, the experimental measurements at $120 \mathrm{kVp}$ were repeated by a completely independent team using different Gammex RMI 467 and CIRS 062M phantoms, a different CT scanner (SOMATOM Confidence RT Pro, Siemens), a different scanning protocol (120 kVp, $225 \mathrm{mAs}$, I30 reconstruction kernel with strength $3,350 \mathrm{~mm}$ field of view, $3 \mathrm{~mm}$ slice thickness) and an independent programming of the stoichiometric fit. In all these cases, the results of these experiments led to equivalent conclusions to those derived from the experimental measurements described above. Thus, only the results of the original experiment are reported here. We also investigated, when possible, the effect of changing the position of the inserts in the calibration phantom, as well as the effect of different reconstruction algorithms. Both effects were found to be of "second-order", i.e. the small differences in CT numbers (due to different insert position and/or reconstruction algorithm) did not alter the conclusions of this work. Slice thickness has been reported to have a negligible effect on the stoichiometric CT calibration curve (Ainsley \& Yeager 2014) and it was therefore not investigated here. The implementation of the stoichiometric fit-i.e. three (Schneider et al. 1996) vs two (Schneider et al. 2000) fitting parameters and minimization algorithm to solve equation (A.22) - was found to have an impact on the resulting $k$-parameters, but it also did not alter the conclusions of this work - unless physically meaningless $k$-values would have been allowed. 


\subsection{Comparison with the 'two-step' SECT calibration method}

Finally, based on the close relationship between the relative stopping power and the relative electron density (A.19) (Schneider et al. 1996), we studied a two-step approach to construct a HU-to-RSP look-up table for proton therapy treatment planning. This two-step method consists of:

1. A HU-to- $\rho_{e}$ LUT based on tissue-substitutes, so that CT numbers can be measured experimentally.

2. A $\rho_{e}$-to-RSP LUT based on biological tissues.

The combination of both LUTs results in the final HU-to-RSP LUT for proton therapy treatment planning. This work compared the two-step method - as a matter of fact step \#1-with the stoichiometric method.

\section{Results and discussion}

\subsection{Theoretical evaluation of tissue-substitutes}

The whole stoichiometric method, as described by Schneider et al. (1996), is based on the assumption that equation (A.7) is correct. If this is the case, section Appendix A shows that the CT number of a given tissue may be expressed as the sum of three terms (A.17): a Compton scattering term proportional to $\rho_{e}$, a Rayleigh scattering term proportional to $\mathcal{Z}_{\mathrm{R}}^{2.86}$ and a photoelectric effect term proportional to $\mathcal{Z}_{\mathrm{p}}^{4.62}$. On the other hand, the relative stopping power is proportional to $\rho_{e}$ (A.19). Thus, a HU-to-RSP CT

calibration curve will have a $x$-axis proportional to $\rho_{e}, \mathcal{Z}_{\mathrm{R}}^{2.86}$ and $\mathcal{Z}_{\mathrm{p}}^{4.62}$; and a $y$-axis proportional to $\rho_{e}$.

Figure 1 shows, for different biological tissues and tissue-substitutes, the relative electron density (A.4) as a function of $\mathcal{Z}_{\mathrm{R}}$ and $\mathcal{Z}_{\mathrm{p}}$ (A.11). It can be seen that Gammex tissue-substitutes seem to agree better with biological tissues than CIRS and Catphan tissue-substitutes in terms of Rayleigh scattering and photoelectric effect.

To quantify this effect on the resulting CT numbers - and thus on the resulting HU-to-RSP look-up table - figure 2 shows, for different biological tissues and tissuesubstitutes, the RSP as a function of the calculated CT number for two CT scans at 80 and $140 \mathrm{kVp}$. As expected from figure 1, figure 2 confirms that Gammex tissue-substitutes agree better with biological tissues than CIRS and Catphan tissuesubstitutes, within the whole energy range relevant to computed tomography.

A second conclusion from figure 2 is that a HU-to-RSP look-up table based on biological tissues (i.e. supposedly accurate in patients) will never yield an accurate prediction of the RSP of CIRS and Catphan bone tissue-substitutes (i.e. those with RSP $\geqslant 1.1$. Again this statement is only true provided that equation (A.7), and therefore (A.17), are correct. 
Figure 1: Relative electron density as a function of $\mathcal{Z}_{\mathrm{R}}$ (top) and $\mathcal{Z}_{\mathrm{p}}$ (bottom), for different biological tissues (White et al. 1992) and tissue-substitutes (Gammex, CIRS, Catphan).
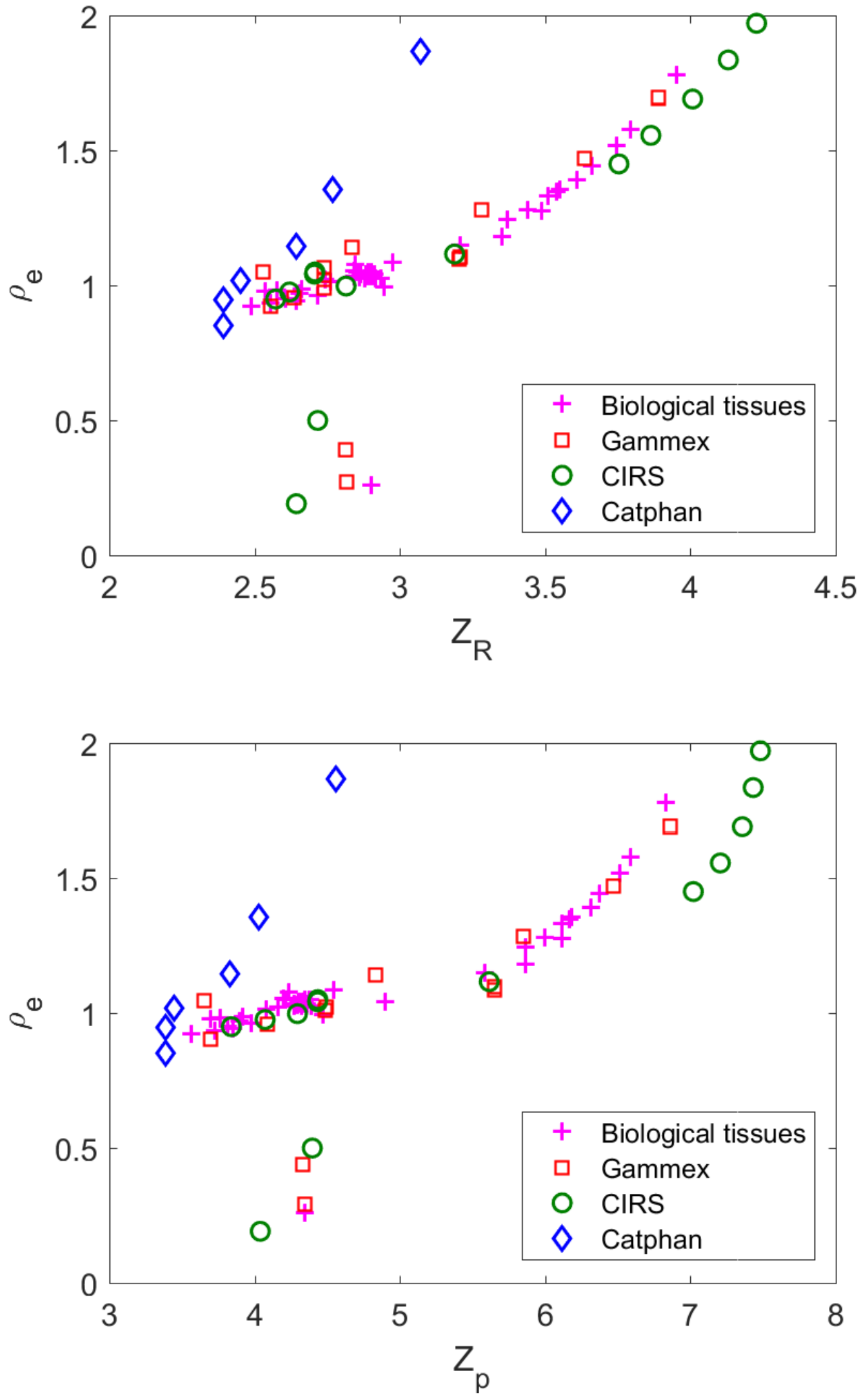
Figure 2: Relative stopping power as a function of the calculated CT number for biological tissues (White et al. 1992) and tissue-substitutes (Gammex, CIRS, Catphan). $\mathrm{CT}$ numbers are calculated using $k$-values representative of $80 \mathrm{kVp}$ (top) and $140 \mathrm{kVp}$ (bottom) x-ray spectra.
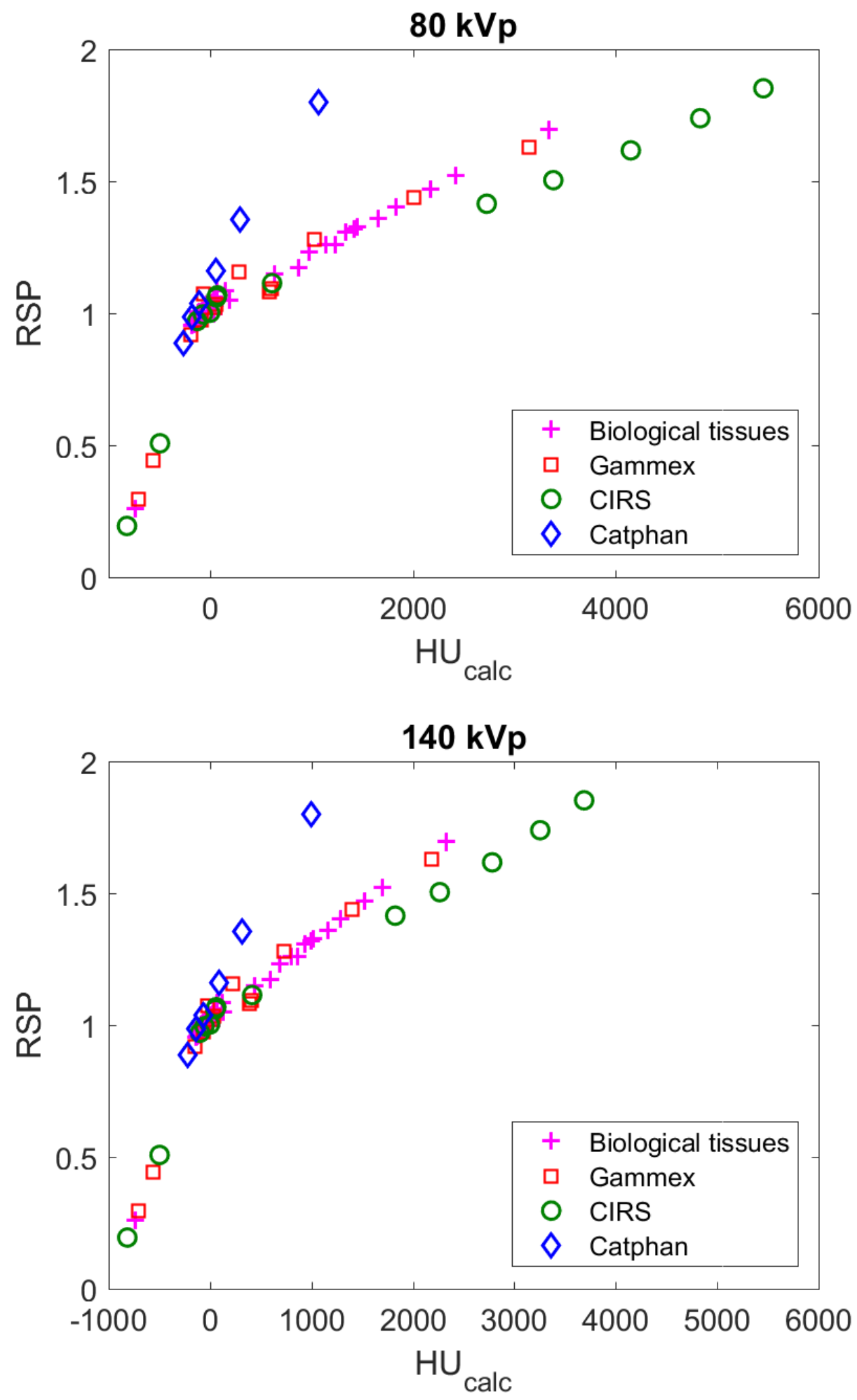
Table 2: Fitting parameters $\left(k_{1}, k_{2}\right)$, covariance matrix $(\Sigma)$ and root-mean-square error of the predicted CT numbers $\left(\mathrm{RMSE}_{\mathrm{HU}}\right)$ obtained with different sets of tissuessubstitutes (Gammex, CIRS and Catphan).

\begin{tabular}{lllllll}
\hline & $k_{1}$ & $k_{2}$ & $\Sigma_{k_{1}, k_{1}}$ & $\Sigma_{k_{2}, k_{2}}$ & $\Sigma_{k_{1}, k_{2}}$ & RMSE $_{\mathrm{HU}}$ \\
\hline Gammex & $5.3 \cdot 10^{-4}$ & $2.3 \cdot 10^{-5}$ & $1.1 \cdot 10^{-6}$ & $1.4 \cdot 10^{-11}$ & $-3.9 \cdot 10^{-9}$ & 20 \\
CIRS & $2.3 \cdot 10^{-4}$ & $1.8 \cdot 10^{-5}$ & $2.0 \cdot 10^{-7}$ & $1.6 \cdot 10^{-12}$ & $-5.6 \cdot 10^{-10}$ & 8 \\
Catphan & $3.8 \cdot 10^{-4}$ & $5.7 \cdot 10^{-5}$ & $6.2 \cdot 10^{-6}$ & $1.4 \cdot 10^{-9}$ & $-9.5 \cdot 10^{-8}$ & 5 \\
\hline
\end{tabular}

Figure 3: Quality of the stoichiometric fits obtained with different sets of tissuesubstitutes (Gammex, CIRS and Catphan). For each set of tissue-substitutes, CT numbers calculated using the $k$-parameters in table 2 are plotted as a function of the measured CT numbers (table 1). Uncertainty bars correspond to two standard deviations. The dotted line indicates a perfect agreement. The inset plot zooms in on the soft tissues.

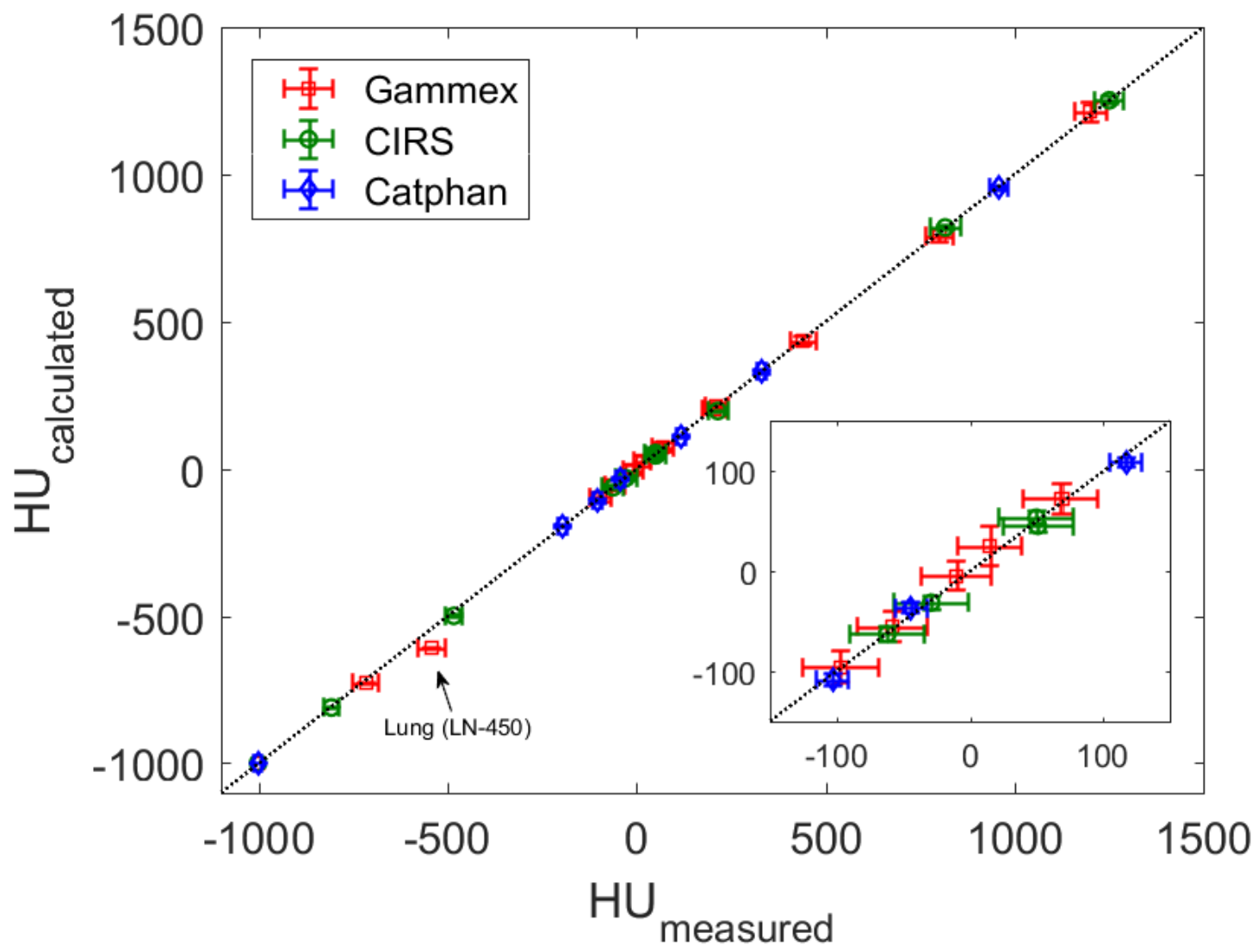




\subsection{Experimental evaluation of the stoichiometric method}

Table 2 shows the fitting parameters $\left(k_{1}, k_{2}\right)$ obtained with the different sets of tissuesubstitutes, together with the covariance matrix of the fit and root-mean-square error of the predicted CT numbers. Figure 3 shows, for each set of tissue-substitutes, the agreement between the CT numbers calculated using the $k$-parameters in table 2 and the measured CT numbers (table 1). It can be seen that, except for one tissuesubstitute (Gammex lung LN-450), calculated and measured CT numbers agree within two standard deviations or better. Nevertheless, the estimated $k$-parameters in table 2 differ notably between the stoichiometric fits obtained with the different sets of tissuesubstitutes. That is, these results seem to suggest that the $k$-parameters not only depend on the CT scanner - equations (A.14) and (A.21) — as initially hypothesized, but also on the tissue-substitutes used for calibration. It should be noted, however, that the three CT calibration phantoms differ (inevitably) in size, so part of the differences in the $k$-parameters might also be due to a slightly different beam hardening.

To assess the impact of these different $k$-parameters on the calculated $\mathrm{CT}$ numbers for biological tissues and thus on the HU-to-RSP look-up table, figure 4 shows the different stoichiometric CT calibration curves-based on ICRU 46 adult biological tissues - obtained with the different $k$-values in table 2 . On the one hand, figure 4 shows that the CT calibration curve obtained with the Catphan materials agrees, within two standard deviations (i.e. $p<0.05$ ), with the curves obtained with the Gammex and CIRS tissue-substitutes - note that, for the sake of clarity, the uncertainty bars in figure 4 only show one standard deviation. That is, the large discrepancy between the Catphan curve and the other two curves is not statistically significant. Nevertheless, the large uncertainty in the calculated CT numbers of biological tissues and, thus, the large uncertainty in the resulting HU-to-RSP look-up table, makes the use of the stoichiometric method in combination with the Catphan phantom unacceptable in clinical practice. Note that these results disagree with the statement "tissue substitutes do not necessarily have to be very tissue-like. It is possible to choose e.g. Lucite, Teflon, Delrin etc." (Schneider et al. 1996).

On the other hand, figure 4 also shows that the differences between the CT calibration curves obtained with the Gammex and CIRS tissue-substitutes are statistically significant $(p<0.05)$ for most bone tissues ( $\mathrm{HU} \geqslant 200)$. That is, figure 4 shows that the stoichiometric CT calibration method depends on the elemental composition of the tissue-substitutes used in the CT characterization. These results disagree with the work of Ainsley \& Yeager (2014), who concluded that Gammex and CIRS tissue-substitutes yielded consistent stoichiometric CT calibration curves based on maximum RSP differences of approximately $1.5 \%$ at $\mathrm{HU}=1500$. The authors, however, did not report an uncertainty estimate on their calculated CT numbers, so it is impossible to conclude whether their reported differences were statistically significant or not.

In particular, figure 4 shows that the differences in relative stopping power between 
Figure 4: Stoichiometric CT calibration curves resulting from the characterization of the same CT scanning protocol with different sets of tissue-substitutes (Gammex, CIRS and Catphan). All curves are built using the same set of biological tissues (White et al. 1992). The uncertainty bars correspond to one standard deviation - if not shown, they are smaller than the marker size. The inset plot zooms in on the soft tissues.

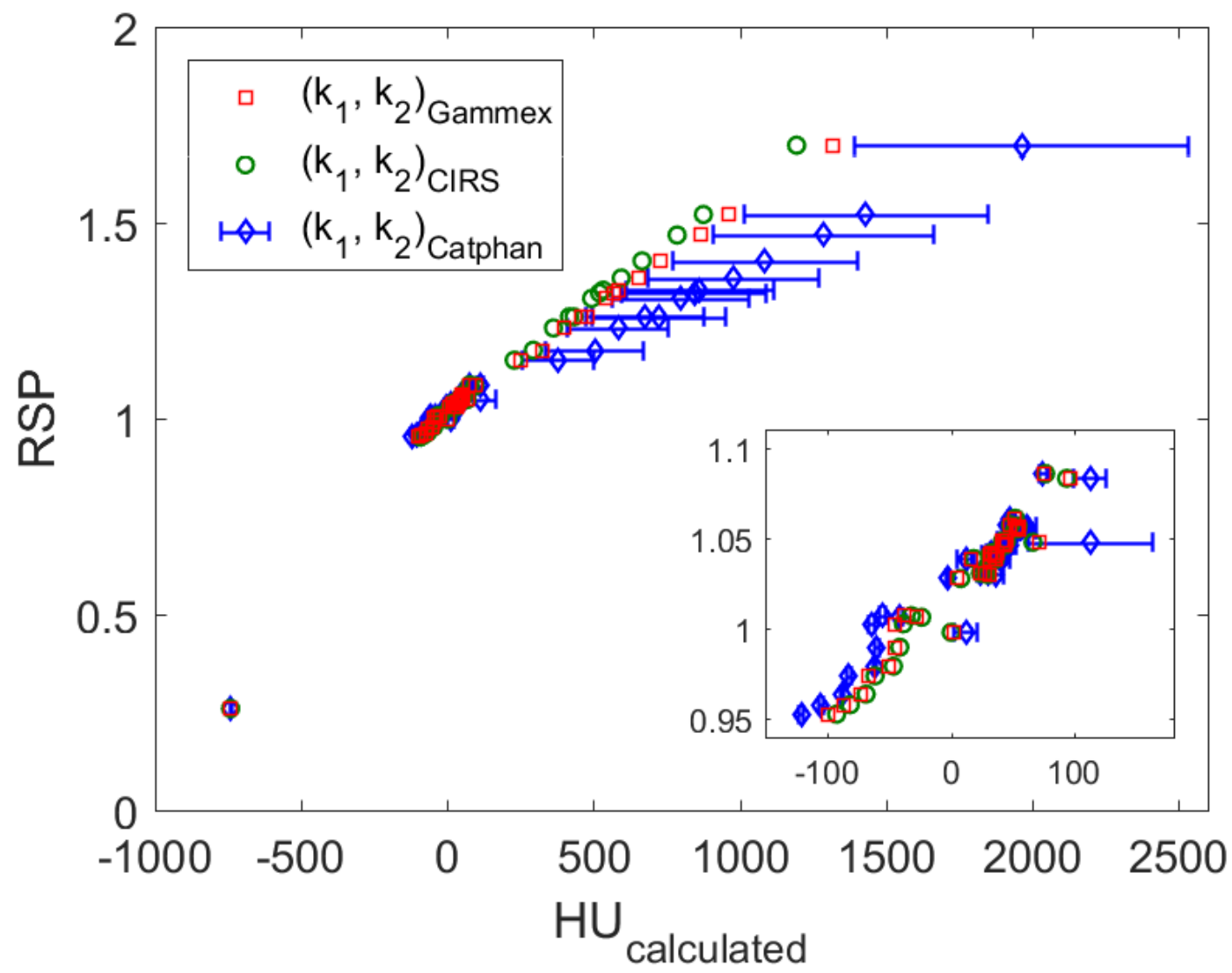

Gammex and CIRS curves range from approximately $1 \%$ for soft bone (HU 200) to about $3.5 \%$ for cortical bone (HU 1200). This could be regarded as an additional source of uncertainty in proton range (Paganetti 2012, Yang et al. 2012) for bone tissues, due to the dependence of the stoichiometric method on the tissue-substitutes used to characterize the CT scanner.

Finally, it is worth mentioning that the use of a different $I_{\mathrm{w}}$-value (than $I_{\mathrm{w}}=78 \mathrm{eV}$ ) would simply rescale up or down all the CT calibration curves in figure 2 and figure 4, but it would not alter the differences between them and, thus, it would not alter the conclusions drawn so far. Likewise, the use of a different proton kinetic energy (than $100 \mathrm{MeV}$ ) would also stretch or compress (along the $y$-axis) the CT calibration curves in figure 2 and figure 4 , but it would maintain the differences between them and, thus, it would not alter either the conclusions drawn so far. 
Figure 5: Relative electron density as a function of CT numbers for biological tissues and tissue-substitutes. The disconnected symbols $(\times, *)$ correspond to tissue-substitutes (Gammex and CIRS) with experimentally measured CT numbers. The connected symbols $(\square, \circ)$ correspond to biological tissues (White et al. 1992) with CT numbers calculated using the different $k$-parameters in table 2 . The uncertainty bars correspond to one standard deviation - if not shown, they are smaller than the marker size. The inset plot zooms in on the soft tissues.

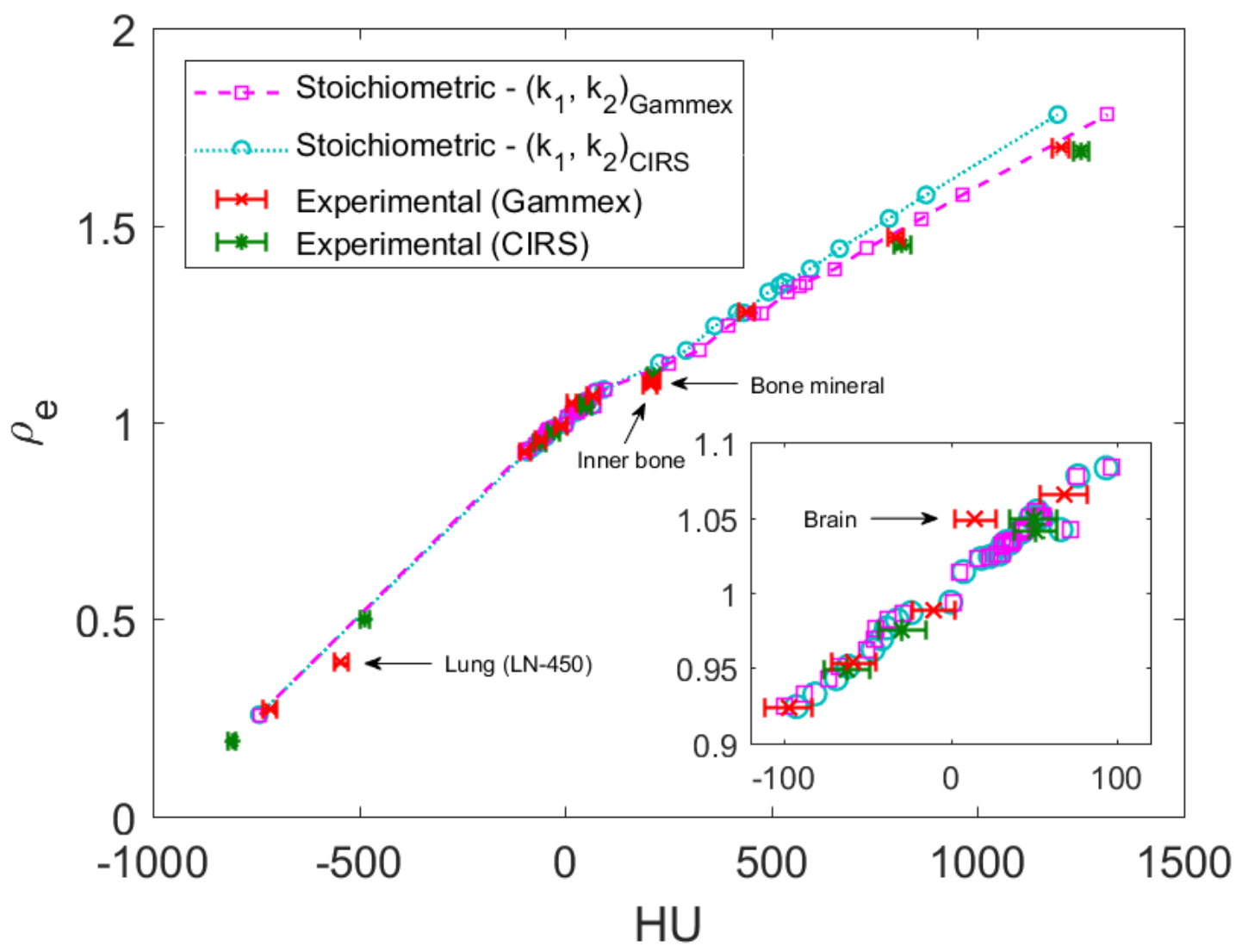

\subsection{Comparison with the 'two-step' SECT calibration method}

Finally, figure 5 shows a comparison between the HU-to- $\rho_{e}$ LUTs obtained experimentally using tissue-substitutes and those obtained using the stoichiometric method. It shows that, when using the Gammex tissue-substitutes for CT calibration, the stoichiometric method becomes unnecessary. That is, the HU-to- $\rho_{e}$ LUT resulting from the stoichiometric method is equal to the one that could be obtained, leaving few tissue-substitutes aside (lung LN-450, brain SR2, inner bone IB3 and bone mineral B200), by simple interpolation of the experimental data. Note, however, that this is not the case for CIRS tissue-substitutes, where experimental and stoichiometric HU-to- $\rho_{e}$ LUT differ significantly for bone tissues.

It should be stressed that this work does not assert which CT calibration curve in 
figure 5 is the most accurate for human tissues - that may only be concluded with some sort of in-vivo range verification (Knopf \& Lomax 2013). This work simply highlights the fact that, in case Gammex tissue-substitutes - or any other tissue-like tissue-substitutes (FitzGerald et al. 2017) - are used for SECT calibration, the stoichiometric method becomes unnecessary and it may be replaced by a much simpler 'two-step' calibration. The latter offers also the advantage of unifying the CT calibration curves for MV-photon beams (Seco \& Evans 2006) and proton beams.

\subsection{Other remarks}

The results of this work are also relevant to recently published research comparing DECT and SECT for proton therapy treatment planning. Figure 5 shows that the stoichiometric SECT calibration curve (typically computed for biological tissues) resulting from experimental measurements in phantom 'A' (e.g. Gammex tissuesubstitutes) will not be able to predict accurately the relative stopping power of bone tissue-substitutes in phantom 'B' (e.g. CIRS). Such experiments, however, have been used in recent publications to report a supposed advantage of DECT over SECT in the prediction of relative stopping powers (Zhu \& Penfold 2016, Michalak et al. 2017). These sort of experiments, however, do not show the limitations of SECT in predicting the relative stopping power of tissue-substitutes, but rather the poor ability of some tissuesubstitutes - namely CIRS and Catphan - to reproduce the CT numbers of biological tissues, for which the stoichiometric SECT calibration curve is typically built. Similar remarks have been made by Wohlfahrt et al. (2016). Note that this comment does not question the demonstrated superiority of DECT over SECT in the prediction of relative stopping powers of biological tissues (Bär et al. 2018b, Möhler et al. 2018, Xie et al. 2018). It just underlines the fact that the improvement of DECT over SECT should be assessed fairly.

It is also important to point out that the conclusions of this work do only apply to the stoichiometric calibration method based on Rutherford et al. (1976) parametrization of the atomic cross-section (A.7), i.e. the stoichiometric method as described in Schneider et al. (1996), Schneider et al. (2000) and used in the vast majority of publications. That is to say that the conclusions of this work may not apply to other stoichiometric calibration methods based on a different parametrization of the atomic/electronic crosssection (Bourque et al. 2014, Lalonde \& Bouchard 2016).

Finally, we believe that the future of proton therapy treatment planning goes towards Monte Carlo dose calculation (Paganetti et al. 2008) and, thus, HU-to-RSP (or HU-to- $\rho_{e}$ ) look-up tables will lose importance in favour of tissue segmentation strategies (Schneider et al. 2000, Hünemohr et al. 2014, Almeida et al. 2018). 


\section{Conclusions}

The results of this work seem to indicate that, unlike originally stated, the stoichiometric method for SECT calibration yields CT calibration curves which depend on the tissuesubstitutes used for calibration - with differences in relative stopping power important for bone tissues only (up to 3.5\% difference). This dependence on the tissue-substitutes used for calibration could be regarded as an additional source of uncertainty in proton range for bone tissues.

The results of this work also suggest that Gammex tissue-substitutes seem to be a better representative of biological tissues than CIRS materials, within the photon energy range relevant to computed tomography. Furthermore, when tissue-like tissuesubstitutes are used for calibration, it has been shown that the stoichiometric method becomes unnecessary and it may be replaced by a much simpler SECT calibration procedure: the 'two-step' method.

\section{Acknowledgements}

We would like to thank Hugo Bouchard, Ruud Houben and Steven Van Aelst for their advice on statistics and/or variance analysis. CG is also thankful to Kinga Bernatowicz, Marie Cohilis, Martin Janson, Uwe Schneider and Edmond Sterpin for the enriching discussions and/or comments on earlier versions of the manuscript.

\section{Appendix A. Theory}

Appendix A.1. Basic quantities

For a given element, the number of atoms per unit volume is given by

$$
n=\rho \frac{N_{\mathrm{A}}}{A}
$$

where $\rho$ is the mass density, $N_{\mathrm{A}}$ is the Avogadro constant and $A$ is the atomic mass. The electron density (i.e. the number of electrons per unit volume) is

$$
n_{e}=n Z=\rho N_{\mathrm{A}} \frac{Z}{A},
$$

where $Z$ is the atomic number of the given element.

In a mixture or compound the electron density is

$$
n_{e}=\rho N_{\mathrm{A}} \sum_{i} w_{i} \frac{Z_{i}}{A_{i}}
$$

where $w_{i}$ is the weight fraction of the $i$-th element in the mixture or compound. The electron density relative to the one of water, also called relative electron density, is

$$
\rho_{e} \doteq \frac{n_{e}}{n_{e, \mathrm{w}}} .
$$


Appendix A.2. X-ray attenuation coefficient and Hounsfield units

The linear attenuation coefficient of a given element is

$$
\mu(E)=n \sigma(E)
$$

where $\sigma(E)$ is the total atomic cross-section, which is a function of the photon energy $E$. The total atomic cross-section may be written as the sum of cross-sections for the processes contributing to the attenuation. In the energy range of computed tomography,

$$
\sigma(E)=\sigma_{\mathrm{C}}(E)+\sigma_{\mathrm{R}}(E)+\sigma_{\mathrm{p}}(E)
$$

where $\sigma_{\mathrm{C}}(E)$ is the atomic cross-section for Compton scattering, $\sigma_{\mathrm{R}}(E)$ is the atomic cross-section for Rayleigh scattering and $\sigma_{\mathrm{p}}(E)$ is the atomic cross-section for photoelectric effect. To a certain level of accuracy, the atomic cross-section may be parametrized as a function of the atomic number (Rutherford et al. 1976, Jackson \& Hawkes 1981)

$$
\sigma(E, Z)=k_{\mathrm{C}}(E) Z+k_{\mathrm{R}}(E) Z^{2.86}+k_{\mathrm{p}}(E) Z^{4.62}
$$

where

$$
k_{\mathrm{C}}(E)=\sigma_{\mathrm{C}}(E) / Z
$$

is the electronic cross-section (in barns) for Compton scattering, and the Rayleigh and photoelectric coefficients may be parametrized as a function of the energy (in keV) as (Rutherford et al. 1976)

$$
\begin{aligned}
k_{\mathrm{R}}(E) & =2.80 E^{-2.02} \\
k_{\mathrm{p}}(E) & =20.64 E^{-3.28} .
\end{aligned}
$$

In a mixture, the linear attenuation coefficient is

$$
\mu(E)=\sum_{i} n_{i} \sigma_{i}(E)=\rho N_{\mathrm{A}} \sum_{i} \frac{w_{i}}{A_{i}} \sigma_{i}(E)
$$

Using (A.7), it follows that

$$
\mu(E)=\rho N_{\mathrm{A}} \sum_{i} \frac{w_{i}}{A_{i}}\left[k_{\mathrm{C}}(E) Z_{i}+k_{\mathrm{R}}(E) Z_{i}^{2.86}+k_{\mathrm{p}}(E) Z_{i}^{4.62}\right]
$$

and, defining

$$
\begin{aligned}
& \mathcal{Z}_{\mathrm{C}} \doteq \sum_{i} \frac{w_{i}}{A_{i}} Z_{i} \\
& \mathcal{Z}_{\mathrm{R}} \doteq\left(\sum_{i} \frac{w_{i}}{A_{i}} Z_{i}^{2.86}\right)^{1 / 2.86} \\
& \mathcal{Z}_{\mathrm{p}} \doteq\left(\sum_{i} \frac{w_{i}}{A_{i}} Z_{i}^{4.62}\right)^{1 / 4.62},
\end{aligned}
$$


it may be expressed as

$$
\mu(E)=\rho N_{\mathrm{A}}\left[k_{\mathrm{C}}(E) \mathcal{Z}_{\mathrm{C}}+k_{\mathrm{R}}(E) \mathcal{Z}_{\mathrm{R}}^{2.86}+k_{\mathrm{p}}(E) \mathcal{Z}_{\mathrm{p}}^{4.62}\right] .
$$

In computed tomography, the effective linear attenuation coefficient may be expressed as

$$
\mu=\int f(E) \mu(E) \mathrm{d} E,
$$

where $f(E)$ is a normalized (i.e. $\int f(E) \mathrm{d} E=1$ ) function which accounts for the polychromatic spectrum of the x-ray source and the energy-dependent response of the radiation detector. Defining in a similar way

$$
k_{i}=\int f(E) k_{i}(E) \mathrm{d} E,
$$

it follows from (A.12) and (A.14) that the effective linear attenuation coefficient (A.13) may be parametrized as

$$
\mu=\rho N_{\mathrm{A}}\left(k_{\mathrm{C}} \mathcal{Z}_{\mathrm{C}}+k_{\mathrm{R}} \mathcal{Z}_{\mathrm{R}}^{2.86}+k_{\mathrm{p}} \mathcal{Z}_{\mathrm{p}}^{4.62}\right) .
$$

Finally, the CT number, or Hounsfield Unit (HU), is defined as (Boone et al. 2012)

$$
\mathrm{HU}=\left(\frac{\mu}{\mu_{\mathrm{w}}}-1\right) \times 1000,
$$

where $\mu$ is the effective linear attenuation coefficient of a given material and $\mu_{\mathrm{w}}$ is that of water. Using (A.3) and (A.15), it follows that the CT number may be expressed as

$$
\mathrm{HU}=K_{\mathrm{C}} \rho_{e}+K_{\mathrm{R}} \mathcal{Z}_{\mathrm{R}}^{2.86}+K_{\mathrm{p}} \mathcal{Z}_{\mathrm{p}}^{4.62}
$$

where $K_{\mathrm{C}}, K_{\mathrm{R}}$ and $K_{\mathrm{p}}$ are proportionality constants. That is, the CT number may be expressed as the sum of three terms: (i) a Compton scattering term which is proportional to the relative electron density $\left(\rho_{e}\right)$, (ii) a Rayleigh scattering term which is proportional to $\mathcal{Z}_{\mathrm{R}}^{2.86}$ and (iii) a photoelectric effect term which is proportional to $\mathcal{Z}_{\mathrm{p}}^{4.62}$.

\section{Appendix A.3. Proton stopping power}

The proton electronic stopping power of a given element or mixture may be expressed as (Berger et al. 1993)

$$
S_{\mathrm{el}}=n_{e} \frac{4 \pi e^{4}}{m_{e} c^{2}} \beta^{2} L(\beta)
$$

where $e$ is the charge of the electron, $m_{e} c^{2}$ is its rest energy, $\beta$ is the velocity of the proton in units of speed of light and $L(\beta)$ is the stopping number. Using Bethe's formulation of the stopping number (Berger et al. 1993), and neglecting both shell and density effect corrections, the proton electronic stopping power relative to the one of water-i.e. the relative stopping power (RSP) — may be approximated as (Schneider et al. 1996)

$$
\mathrm{RSP} \doteq \frac{S_{\mathrm{el}}}{S_{\mathrm{el}, \mathrm{w}}}=\rho_{e} \frac{\ln \left(2 m_{e} c^{2} \beta^{2}\right)-\ln \left(1-\beta^{2}\right)-\beta^{2}-\ln I}{\ln \left(2 m_{e} c^{2} \beta^{2}\right)-\ln \left(1-\beta^{2}\right)-\beta^{2}-\ln I_{\mathrm{w}}} \equiv K_{\mathrm{S}} \rho_{\mathrm{e}} .
$$

where $I$ is the mean excitation energy of the medium. That is, the relative stopping power is directly proportional to the relative electron density $\left(\rho_{e}\right)$. 
Appendix A.4. Stoichiometric fit

From (A.11) and (A.15) it follows that the ratio of the effective linear attenuation coefficients of a given material and the one of water may be expressed as (Schneider et al. 2000)

$$
\frac{\mu}{\mu_{\mathrm{w}}}=\frac{\rho}{\rho_{\mathrm{w}}} \frac{\sum_{i} \frac{w_{i}}{A_{i}}\left(Z_{i}+k_{1} Z_{i}^{2.86}+k_{2} Z_{i}^{4.62}\right)}{\frac{w_{\mathrm{H}}}{A_{\mathrm{H}}}\left(1+k_{1}+k_{2}\right)+\frac{w_{\mathrm{O}}}{A_{\mathrm{O}}}\left(8+k_{1} 8^{2.86}+k_{2} 8^{4.62}\right)},
$$

where

$$
k_{1} \doteq \frac{k_{\mathrm{R}}}{k_{\mathrm{C}}} \quad \text { and } \quad k_{2} \doteq \frac{k_{\mathrm{p}}}{k_{\mathrm{C}}} .
$$

The fitting parameters $k=\left(k_{1}, k_{2}\right)$ characterize the $\mathrm{CT}$ scanner (both the x-ray spectrum and the detector energy response) for a given scanning protocol. These parameters may be determined by minimizing the expression (Schneider et al. 2000)

$$
\sum_{j}\left[\left(\frac{\mu}{\mu_{\mathrm{w}}}\left(k_{1}, k_{2}\right)\right)_{j}-\left(\frac{\mathrm{HU}}{1000}+1\right)_{j}\right]^{2},
$$

where $j$ runs over all tissue-substitutes used in the CT characterization. Once the CT scanner is characterized - i.e. once the $k$-values are determined - one can calculate the CT number of any tissue of known elemental composition $\left(\rho, w_{i}, Z_{i}\right)$, using equations (A.16) and (A.20).

\section{Appendix B. Variance analysis}

Let us define the reduced $\mathrm{HU}$

$$
u=\frac{\mu}{\mu_{\mathrm{w}}}
$$

which, according to (A.20), is a function of the $k$-parameters $\left(k_{1}, k_{2}\right)$, i.e. $u=u(k)$.

According to the delta method, the variance of $u$ is

$$
\operatorname{Var}(u(k))=\nabla_{k}(u)^{\mathrm{T}} \Sigma_{k} \nabla_{k}(u),
$$

where

$$
\nabla_{k}(u)=\left(\frac{\partial u}{\partial k_{1}}, \frac{\partial u}{\partial k_{2}}\right)
$$

and $\Sigma_{k}$ is the covariance matrix of $k$. Finally the standard deviation of HU (A.16) is

$$
\sigma_{\mathrm{HU}}=1000 \sqrt{\operatorname{Var}(u)} .
$$

\section{References}

C. G. Ainsley \& C. M. Yeager (2014). 'Practical considerations in the calibration of CT scanners for proton therapy'. Journal of Applied Clinical Medical Physics 15(3):20220 . 
I. P. Almeida, et al. (2018). 'Monte Carlo proton dose calculations using a radiotherapy specific dual-energy CT scanner for tissue segmentation and range assessment'. Physics in Medicine and Biology 63(11):115008.

E. Bär, et al. (2018a). 'Optimized $I$-values for use with the Bragg additivity rule and their impact on proton stopping power and range uncertainty'. Physics in Medicine and Biology 63(16):165007.

E. Bär, et al. (2017). 'The potential of dual-energy CT to reduce proton beam range uncertainties'. Medical Physics 44(6):2332-2344.

E. Bär, et al. (2018b). 'Experimental validation of two dual-energy CT methods for proton therapy using heterogeneous tissue samples'. Medical Physics 45(1):48-59.

M. J. Berger, et al. (1993). 'Stopping Powers and Ranges for Protons and Alpha Particles'. ICRU Report 49, International Commission on Radiation Units and Measurements.

M. J. Berger, et al. (1984). 'Stopping Powers for Electrons and Positrons'. ICRU Report 37, International Commission on Radiation Units and Measurements.

J. M. Boone, et al. (2012). 'Radiation dose and image-quality assessment in computed tomography. ICRU Report 87'. Journal of the ICRU 12(1).

A. E. Bourque, et al. (2014). 'A stoichiometric calibration method for dual energy computed tomography'. Physics in Medicine and Biology 59:2059-88.

E. A. De Kock \& A. N. Schreuder (1996). 'Comments 'The calibration of CT Hounsfield units for radiotherapy treatment planning'. Physics in Medicine and Biology 41:15246.

V. De Smet, et al. (2018). 'Reassessment of stopping power ratio uncertainties caused by mean excitation energies using a water-based formalism'. Medical Physics 45(7):33613370.

P. F. FitzGerald, et al. (2017). 'Liquid tissue surrogates for X-ray and CT phantom studies'. Medical Physics 44(12):6251-6260.

N. Hünemohr, et al. (2014). 'Tissue decomposition from dual energy CT data for MC based dose calculation in particle therapy'. Medical Physics 41(6):061714.

D. F. Jackson \& D. J. Hawkes (1981). 'X-ray attenuation coefficients of elements and mixtures'. Physics Reports 70(1):169-233.

A.-C. Knopf \& A. Lomax (2013). 'In vivo proton range verification: a review'. Physics in Medicine and Biology 58(15):R131-60.

A. Lalonde \& H. Bouchard (2016). 'A general method to derive tissue parameters for Monte Carlo dose calculation with multi-energy CT'. Physics in Medicine and Biology 61(22):8044.

G. Michalak, et al. (2017). 'A comparison of relative proton stopping power measurements across patient size using dual- and single-energy CT'. Acta Oncologica 56(11):1465-1471. 
C. Möhler, et al. (2018). 'Experimental verification of stopping-power prediction from single- and dual-energy computed tomography in biological tissues'. Physics in Medicine and Biology 63(2):025001.

H. Paganetti (2012). 'Range uncertainties in proton therapy and the role of Monte Carlo simulations'. Physics in Medicine and Biology 57(11):R99-117.

H. Paganetti, et al. (2008). 'Clinical implementation of full Monte Carlo dose calculation in proton beam therapy'. Physics in Medicine and Biology 53(17):4825-53.

R. A. Rutherford, et al. (1976). 'Measurement of effective atomic number and electron density using an EMI scanner'. Neuroradiology 11(1):15-21.

U. Schneider, et al. (1996). 'The calibration of CT Hounsfield units for radiotherapy treatment planning'. Physics in Medicine and Biology 41:111-24.

W. Schneider, et al. (2000). 'Correlation between CT numbers and tissue parameters needed for Monte Carlo simulation of clinical dose distributions'. Physics in Medicine and Biology 45:459-78.

J. Seco \& P. M. Evans (2006). 'Assessing the effect of electron density in photon dose calculations'. Medical Physics 33(2):540-552.

S. M. Seltzer, et al. (2011). 'Fundamental Quantities and Units for Ionizing Radiation (Revised). ICRU Report No. 85'. Journal of the ICRU 11(1).

S. M. Seltzer, et al. (2016). 'Key data for ionizing radiation dosimetry: measurement standards and applications. ICRU Report 90'. Journal of the ICRU 14(1).

V. T. Taasti, et al. (2018). 'Inter-centre variability of CT-based stopping-power prediction in particle therapy: Survey-based evaluation'. Physics and Imaging in Radiation Oncology 6:25-30.

B. van der Heyden, et al. (2018). 'VOXSI: A vowelised single- and dual-energy CT scenario generator for quantitative imaging'. Physics and Imaging in Radiation Oncology 6:47-52.

F. Verhaegen \& S. Devic (2005). 'Sensitivity study for CT image use in Monte Carlo treatment planning'. Physics in Medicine and Biology 50(5):937-46.

D. R. White, et al. (1992). 'Photon, Electron, Proton and Neutron Interaction Data for Body Tissues'. ICRU Report 46, International Commission on Radiation Units and Measurements.

P. Wohlfahrt, et al. (2016). 'Comment on: Dosimetric comparison of stopping-power calibration with dual-energy CT and single-energy CT in proton therapy treatment planning [Med. Phys. 43(6), 2845-2854 (2016)]'. Medical Physics 44(10):5533-5536.

Y. Xie, et al. (2018). 'Ex vivo validation of a stoichiometric dual energy CT proton stopping power ratio calibration'. Physics in Medicine and Biology 63(5):055016.

M. Yang, et al. (2012). 'Comprehensive analysis of proton range uncertainties related to patient stopping-power-ratio estimation using the stoichiometric calibration'. Physics in Medicine and Biology 57(13):4095. 
J. Zhu \& S. N. Penfold (2016). 'Dosimetric comparison of stopping power calibration with dual-energy CT and single-energy CT in proton therapy treatment planning'. Medical Physics 43:2845-54. 